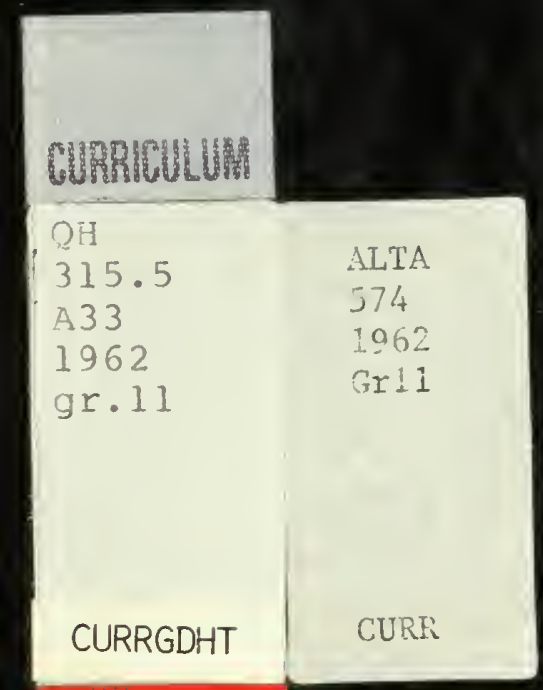




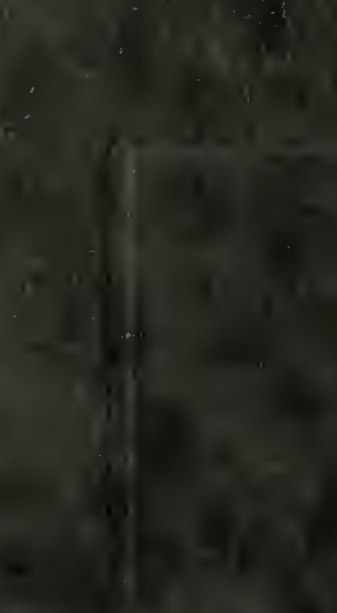




\section{BIOLOGY 20}

(Interim Outline)

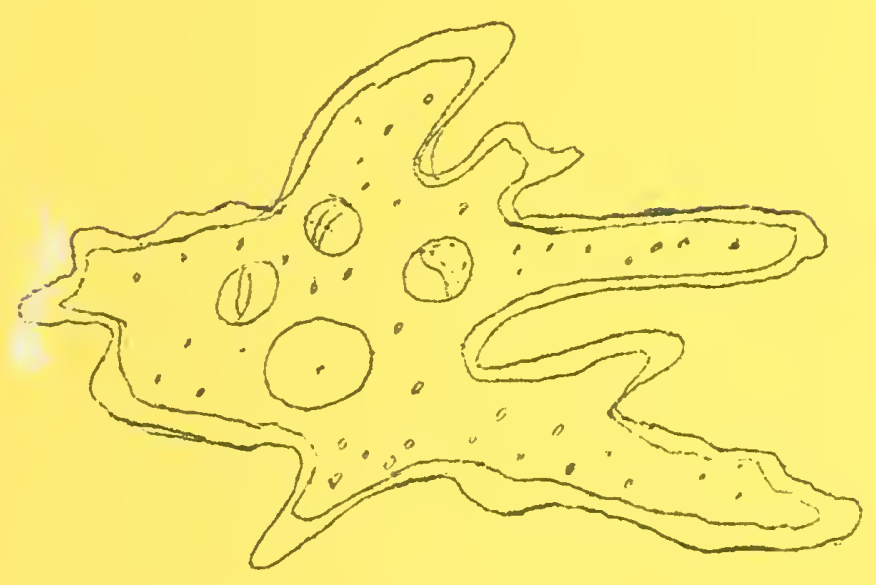

DEPARTMENT OF EDUCATION 
Digitized by the Internet Archive in 2012 with funding from University of Alberta Libraries 
Texts

I. MODERN BIOLOGY; Moon, Otto and Towle; Holt, Rhinehart \& Winston, Inc.

2. RENEWABIE RESOURCES OF ALBERTA; Department of Education

Aims and Objectives

1. To ensure an acquaintance with a wide variety of organisms and thereby provide a background for a detailed study of biological phenomena.

2. To trace the development of organisms from the simple to the complex.

3. To foster an appreciation of the interdependence of living things and the importance of the conservation of renewable natural resources.

4. To promote an understanding of the use of the scientific method in biology through field observation and laboratory exercise.

Laboratory Work

The stress in this course should be on first hand observation of organisms and their behaviour. Forty exercises have been outlined in the laboratory manual. Ideally, all of these should be done by the students during the year. Two periods out of five should be allocated for laboratory work. Every effort should be made to secure equipment and materials for this purpose. A good deal of the material can be collected and preserved for use in the laboratory. Where possible, laboratory work should articulate with the corresponding topic covered in class periods.

Time Allotment

It is suggested that the section on botany be completed in the first term and the remaining sections be completed during the second term.

Evaluation

It is recommended that in evaluating progress of students, $30 \%-50 \%$ be derived from laboratory work such as laboratory reports, exercise books and practical tests. ultimately, $50 \%$ of the mark of any student should be based on laboratory work.

\section{Course Outline}

All page references are to the text, MODERN BIOLOGY by Moon et al. The recommended laboratory exercises refer to the exercise number in the manual. 

nit I: THE SCIENTIFIC STUDY OF LIVING THINGS (Ch. 1-7)

Topic

Text Reference

Lab. Exercise

ntroduction

Biology defined

Historical

Microscopy

Branches of biology

Properties of life

Basis of life

Development of the

cell theory

Ce? I - murture

Cell types

Cell organization

iffe processes

rods

Review elem. chem.

Classes of foods and

specific role of each
P. I

P. 1 and 2

P. $5-8$

P. $10-11$

Ch. 2 (briefly)

P. $21-22$

3

P. $22-26$

P. 26

P. $28-29$

Ch. 4 (briefly)

Film or film strip on mitosis and meiosis

init II: CLASSIFICATION OF ORGANISMS (Ch. 8)

inis unit involves a brief examination of the classification system, to be applied in jucceeding chapters.

Tilit III: THE FLOWERLESS PLANTS (Ch. 9-12)

Topic

Text Reference

Lab. Exercise

1.?. gee characteristics

$\begin{array}{ll}\text { Closterium } & \text { P. } 91 \\ \text { Spirogyra } & \text { P. } 95-97 \\ \text { Ulothrix (briefly) } & \text { P. } 97-98 \\ \text { Oedogonium (briefly) } & \text { P. } 98-100\end{array}$

6

7 and 8

Sasteria

Description

Form

Conditions for growth

Useful activities

Bacteria and disease

P. 104

P. 105 - 106

P. 107

P. 110 - 111

P. $569-570$ 

Fungi

General characteristics

Bread mold

Penicillium

Yeast
P. $114-115$

P. 115

P. 116

P. $117-118$

P. $127-128$

P. $131-132$
10

11

$\left\{\begin{array}{c}\text { (To be done with } \\ \text { charts }\end{array}\right.$

\section{Topic}

General

The root

Structure

Absorption

The stem

The leaf

The flower

The seed

Defined

Monocot

Dicot

Germination
Text Reference

Ch. 13

Ch. IL

Ch. 15

Ch. 16

Ch. 17

Ch. 18

P. 216

P. 218

P. 217

P. $220-222$
Lab. Exercise

12

13 (demonstration)

14 (demonstration)

15 and 16

17 and 18

(demonstration)

19

20

Lab. Exercise

21

22

23

$$
\begin{aligned}
& \text { P. } 231-233 \\
& \text { P. } 234-236 \\
& \text { P. } 233 \\
& \text { P. } 238-239 ; 569
\end{aligned}
$$

Text Reference

$$
\begin{aligned}
& \text { P. } 214 \\
& P .252 \\
& P .243 \\
& P .244-245 \\
& P .246-248 \\
& P .253-254 \\
& P .255 \\
& P .254
\end{aligned}
$$

P. 254 $\frac{\text { Planarian }}{\text { Tapeworms }}$

Flukes 

Metazoa (continued)

Roundworms
Annelids
P. $256-257$
Earthworm
P. $259-262$

Mollusks (omit internal anatomy) P. $265-268$

Echinoderms

Starfish (omit internal $\quad$ P. 269 - 271

Arthropods

$$
\begin{aligned}
& \text { Characteristics } \\
& \text { Crayfish (external) } \\
& \text { Spider (external) } \\
& \text { Ins ects } \\
& \text { Specialization } \\
& \text { Grasshopper } \\
& \text { General features) } \\
& \text { ventral nerve ) } \\
& \text { cord) P. } 287-291 \\
& \text { open vascular ) } \\
& \text { P. } 273 \\
& \text { P. } 274-278 \\
& \text { P. } 281-282 \\
& \text { P. } 286-287 \\
& \text { system } \\
& \text { Other insects: gen- P. } 294-308
\end{aligned}
$$

Unit VI: VERTEBRATES (ch. 28-33)

\section{Topic}

Characteristics

Importance

Bony fish

External features

Internal anatomy

Amphibians

Characteristics and

representatives

Frog

$$
\begin{aligned}
& \text { External features } \\
& \text { Internal anatomy }
\end{aligned}
$$

Text Reference

Lab. Exercise

P. $322-324$

$$
\begin{aligned}
& \text { P. } 330-332) \\
& P .332-336)
\end{aligned}
$$

Reptiles
Characteristics (no struc- P. 359 tural detail)
Representatives (snakes and P. 365-367, 371

$$
\text { turties ) }
$$

Birds

Characteristics and adapta- P. 357 - 379 (first column) tions for flight

Mammals

Characteristics

Reproduction system

Nervous system

Defence structures
P. 342

P. $347-348$

P. $349-353$
$33-34$

$35-39$ 

Unit VII: ENDOCRINE GLANDS (Ch. LI)

Topic

Definition and importance

Thyroid and metabolism

Pituitary

Adrenal

Pancreas (minimum of detail)
Text Reference

P. 515

P. $516-517$

P. $517-518$

P. 518

P. 519

Lab. Exercise

Unit VIII: HEREDITY (ch. 49-52)

\section{Topic}

Significance of nucleus

Mitosis

Meiosis (omit technical terms; sperm, polar bodies)

Mendel's laws

$\$ \approx x$ determination

Sex linked characteristics

Mutations
Text Reference

Lab. Exercise

P. $609-610$

P. $610-613$

P. $614-616$

40

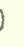

Note: Chapter 51 to be assigned for reading only.

Unit IX: CHANGING LIFE (Ch. 53)

\section{Topic}

Change vs. Origin

Evidence of change

Theories

Lamarck
Weismann
Darwin
DeVries

Text Reference

Lab. Exercise
P. $620-626$

P. $629-631$

P. 651

P. $632-633,654$ 
, 
Microscope - One for every two students in the class

Prepared slides:

Botanical: Closterium

Spirogyra (conjugating)

Root (x-section)

Woody dicot stem $(x-s)$

Alfalfa stem $(x-s)$

Corn stem $(x-s)$

Leaf $(x-s)$

\author{
Zoological: Amoeba (w-m) \\ Hydra $(w-m)$ \\ $\overline{\text { Planarian }(w-m)}$ \\ Tapeworm $(w-m)$ \\ Tapeworm $(x-s)$ \\ Earthworm ( $x-s)$ \\ Onion root tip (mitosis) \\ Whitefish eggs (mitosis)
}

Preserved material:

Botanical:

Zoological: Earthworm

Grasshopper

Perch or pike (may be

taken Iocally and frozen)

Frog

Live material: (much of this may be collected and cultured locally)

Botanical: Elodea (Anacharis)

Slosterium
Zoological: Amoeba

Paramecium

Euglena

Hydra

Planarian

Gold fish

Chemicals:

Methyl cellulose

Carbon disulfide

Methyl blue (stain)

Agar agar (powdered)

Tryptone

Ammonia

Carmine stain

Gentian (crystal) violet (aqueous)

Litmus (dry)

Potassium iodide

Other equipment:

Slides (ordinary and cavity)

Pipettes or eye droppers

Scissors

Dissecting needles

Dialiting membrane
Sulphur (powdered)

Iodine solution (stain)

Glucose

Beef extract

Fehling's $A$ and $B$ or Benedict's sol'n

Potassium permanganate

Dextrose (powdered)

$\mathrm{xy}$ Iol

Ethanol

Acestone

Cover slips

Dissecting trays (waxed)

Forceps

Hydrion pH paper

Glass tubing 
! 
Petri dishes

Inoculating needles

Test tubes (bacteriological)

Hand lenses

Aquaria

Materials which may be secured locally:

Clay

Cork

Potato

Lean beef

Vinegar

Yeast (dry)

Table sugar

Apple juice

Timothy or Red Top Seed

Molasses

Corn seed

Garanium for cuttings
Wax pencil

Bacteriological plugging cotton 4-6 inch sticks for swabs

Battery jars

Beakers

Carrot

Onion

Tomato

Rolled nats

Milk (raw and pasteurized)

Brown sugar

Honey

Corn starch

Radish seeds

Bean seeds

Coleus (green-white)

Twigs of poplar and two others 



\section{DATE DUE SLIP}

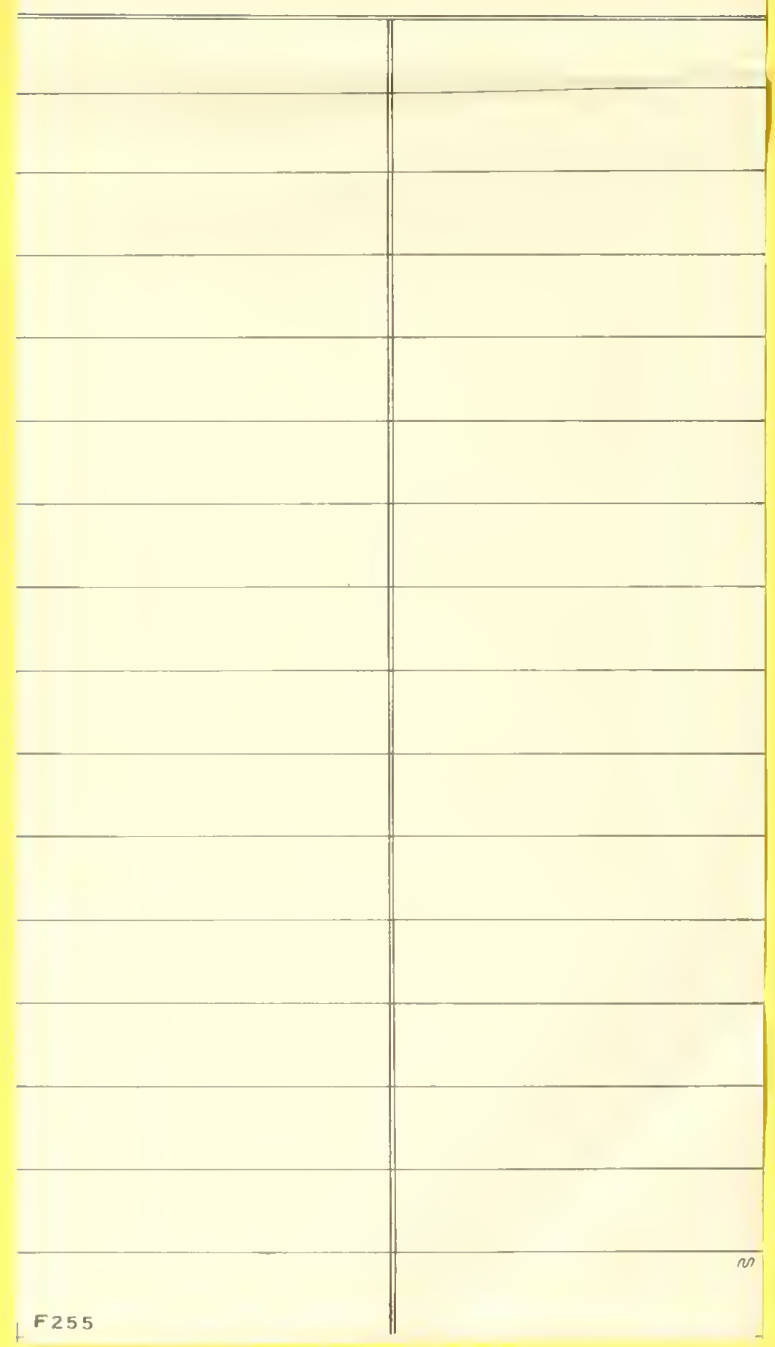


OH 315-5 A33 1962 GR-11

BIOLOGY 20 - /INTERIM OUTLINE --

39841045 CURR HIST
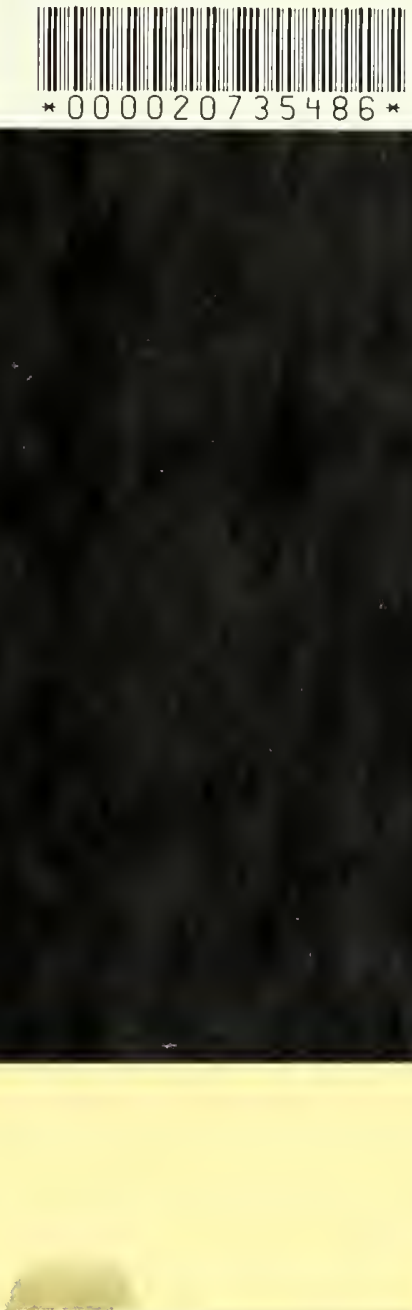

QH 315.5 A33 1962 gr. 11 Biology 20 . -

39841045 CURR HIST

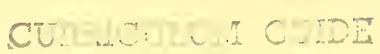

\section{For Reference}

NOT TO BE TAKEN FROM THIS ROOM 
